\title{
The Correlation between Clinical and Pathological Lymph Node Status in Oral Squamous Cell Carcinoma
}

\author{
Evan Roche Rabie ${ }^{1 *}$, Ciska-Mari Schouwstra ${ }^{2}$ \\ ${ }^{1}$ Department of Maxillofacial and Oral Surgery, University of Pretoria, Pretoria Oral Health Center, South Africa \\ ${ }^{2}$ Department of Oral Biology and Oral Pathology, University of Pretoria, Pretoria Oral Health Center, South Africa
}

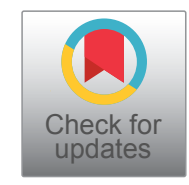

\begin{abstract}
Introduction: The presence of nodal metastasis from OSCC is the single most important prognostic feature. Extranodal extension further decreases survival by approximately half. Clinical evaluation of the nodal basin has been found to correlate poorly with pathological nodal status.

Objectives: Determine the congruency between clinical and pathological nodal status and the incidence of extranodal extension (ENE). Describe the frequency distribution of age, sex, smoking habits, anatomical location, and histology in a South African population.

Methods: Ninety-one consecutive cases of oral squamous cell carcinoma were retrospectively studied and the clinical $(\mathrm{cN})$ and pathological $(\mathrm{pN})$ TNM nodal staging compared using the $8^{\text {th }}$ edition of the AJCC/UICC TNM staging system. Extranodal extension was determined in all patients with OSCC, as well as in the subgroup with clinically nodal negative (cNO) disease. The socio-demographical information was described.

Results: The $\mathrm{cN}$ and $\mathrm{pN}$ were congruent in $41.7 \%(\mathrm{n}=38 / 91)$, upstaged in $35.2 \%(\mathrm{n}=32 / 91)$, and down staged in $23.1 \%$ ( $n=21 / 91$ ) of cases. Twenty-two patients (24.2\%) had pathological evidence of ENE. Males and females constituted $67 \%$ and $33 \%$ of the study sample, respectively. The median age at the time of diagnosis was 57.3 years (range 5-85 years). A smoking history was present in $73.6 \%$, absent in $14.3 \%$, and unknown in $12.1 \%$ of patients. The tongue (38.4\%) and floor of mouth $(29.7 \%)$ were the most common anatomical subsites for primary tumours. Most tumours were conventional squamous cell carcinomas with poorly differentiated grade $(62.6 \%)$
\end{abstract}

Conclusion: There is poor correlation between clinical and pathological nodal status when the $8^{\text {th }}$ TNM staging is used. ENE at the time of diagnosis is common among patients with OSCC.

\section{Keywords}

Oral cancer, Oral squamous cell carcinoma, Clinical nodal staging, Pathological nodal Staging, Extranodal extension, Stage migration

\section{Introduction}

Oral squamous cell carcinoma (OSCC) constitutes up to $90 \%$ of oral cancer diagnoses [1,2]. OSCC has the propensity to demonstrate lymph node metastasis at the time of clinical presentation, conferring a poor prognosis. Approximately one third of patients harbour regional metastasis at time of diagnosis [3-5]. Staging systems play a central role in caring for patients with any malignancy. Patient counselling, treatment planning, prognostication and the judicious design of clinical trials are based on tumour staging. The most widely used staging system is the TNM-staging system published jointly by the American Joint Committee on Cancer/ Union for International Cancer Control (AJCC/UICC), currently in its eight editions [6]. To ensure accurate treatment planning, congruency between clinical and pathological nodal staging is essential. This is particularly relevant in resource constrained environments with restricted access to computed tomography leading to long waiting times, unnecessarily delaying surgical care.

*Corresponding author: Evan Roche Rabie, Department of Maxillofacial and Oral Surgery, University of Pretoria, Pretoria Oral Health Center, Room 2-43, Prinshoff Campus, Pretoria 0002, South Africa, E-mail address: evan.rabie@gmail.com

Accepted: June 11, 2021

Published online: June 14, 2021

Citation: Rabie ER, Schouwstra CM (2021) The Correlation between Clinical and Pathological Lymph Node Status in Oral Squamous Cell Carcinoma. J Oral Cancer Res 4(1):49-56 
The extent of neck dissection offered is dependent on the clinical TNM (CTNM) whereas adjuvant treatment is based on pathological TNM (pTNM). Clinically node negative (cNO) patients who are pathologically upstaged, are at risk of having received inadequate neck dissection leading to residual disease in nodal basins outside the surgical field. It is well established that the presence of nodal metastasis affecting the first-echelon lymph nodes increases the risk of nodal metastasis in distant nodal basins [5]. Hence, the aims of the current study were to determine the congruency between the $\mathrm{CN}$ and $\mathrm{pN}$ staging in a retrospective design. Secondary objectives aimed to establish the frequency of extranodal extension (ENE) in all OSCC patients and in the sub-group of patients staged with cNO disease. The frequency distribution of age, sex, smoking habits, anatomical location, and histology was also described.

\section{Material and Methods}

\section{Study design}

A non-randomised retrospective study of patients diagnosed with oral squamous cell carcinoma (OSCC) and treated with tumour excision and neck dissection, between 1 January 2007 and 31 December 2019, were conducted. A search of information databases at the Department of Oral Pathology and Oral Biology, University of Pretoria, were performed. The search results were cross-referenced to information databases and patient clinical records held in the Department of Maxillofacial and Oral surgery at University of Pretoria, to identify patients diagnosed and treated for OSCC. Patients with a histologic diagnosis of OSCC with clinical records documenting CTNM and postoperative histological reports documenting PTNM, were eligible for inclusion. The clinical staging (CTNM) was determined by inspection and palpation and supplemented with contrast enhanced computed tomography when available and not unduly delaying surgical care. The following patients were excluded. Patients that lacked clinical patient records or a postoperative histological report documenting pTNM. Any neck dissection that did not include at least levels I to III or salvage neck dissections where excluded.

\section{Surgical Protocol}

Standard anaesthetic technique was followed by either nasoendotracheal intubation, endotracheal intubation, or tracheostomy. Standard antiseptic techniques were observed. Patients that presented with no clinical cervical lymphadenopathy (cNO) were offered an elective selective neck dissection including levels I-III. Patients that presented with clinical cervical lymphadenopathy $(\mathrm{cN}+)$ were offered a modified radical neck dissection (MRND) including levels $\mathrm{I}-\mathrm{V}$, with or without preservation of the sternocleidomastoid muscle, internal jugular vein and/or accessory nerve (CNXI).

\section{Histological Assessment and Reporting}

Standard laboratory techniques were used to isolate lymph nodes, document their anatomical location, and tissue blocks were processed to prepare histological slides for light microscopic evaluation by a pathologist. Haematoxylin and eosin (H\&E)-stained $4 \mu \mathrm{m}$ thick sections of harvested lymph nodes were assessed for the presence of metastatic tumour deposits and ENE. Based on the $7^{\text {th }}$ edition (before 2017) or the $8^{\text {th }}$ edition (after 2017) of the AJCC-TNM cancer staging manual, $\mathrm{pN}$-stage was determined and reported. All pathological reports that used the $7^{\text {th }}$ edition of the AJCCTNM criteria for $\mathrm{pN}$ staging were reviewed and graded with the criteria set out in the $8^{\text {th }}$ edition.

\section{Statistical Analysis}

Descriptive statistics were combined with Pearson Chi-square analysis and Fisher Exact Tests to collate the relationships between clinical nodal $(\mathrm{cN})$, pathological nodal $(\mathrm{pN})$ and extranodal extension (ENE). The $\mathrm{cN}$ and $\mathrm{pN}$ were tabulated and compared to determine whether upstaging, downstaging or concordance in staging occurred. The incidence of ENE in all cases of OSCC and the CNO subgroup was assessed and documented as a percentage. Statistical analysis was performed using SPSS (version 27, IBM, New York, United States of America.

\section{Sample Size}

Convenience sampling was employed. All available cases that had primary oral squamous cell carcinoma and had undergone surgical resection of the primary tumour with neck dissection were considered for inclusion. The sample size was sufficient for descriptive purposes, including risk analysis.

\section{Results}

A total of 108 consecutive patients with oral squamous cell carcinoma were considered for inclusion. After application of the inclusion and exclusion criteria 17 patients were excluded, resulting in a final study sample of 91 cases. The mean age of the study population was 57 years (range 5-85) with 61 males $(67 \%)$ and 30 females (33\%). A smoking history was present in $73.6 \%$, absent in $14.3 \%$, and unknown in $12.1 \%$ of patients. The oral tongue was the most frequently affected site with $38,4 \%$ ( $n=35 / 91$ ) followed by the floor of mouth $29.7 \%$ ( $n=$ $27 / 91)$, alveolar ridge, $17.6 \%(n=16 / 91)$, retromolar trigone $9.9 \%(n=9 / 91)$, and buccal mucosa and hard palate with $2.2 \%(n=2 / 91)$, respectively. Conventional SCC was the most common histological subtype, $62.6 \%$ of which were poorly differentiated. Histological variants of SCC were found in 6 cases. Histological differentiation was not available for $9,9 \%$ $(n=9 / 91)$ of cases. The socio-demographical data and tumour factors are summarised in (Table 1).

The prevalence of $\mathrm{cN}$ and $\mathrm{pN}$-staging subgroups are summarised in (Graph 1). At diagnosis 53.8\% ( $n=49 / 91$ ) of patients had no clinical cervical lymphadenopathy (cNO). After surgery $52.7 \%$ ( $n=48 / 91$ ) of patients had no pathological identified nodal metastasis (pNO). A total of $46.2 \%$ ( $n=42 / 91)$ of patients presented with clinical cervical lymphadenopathy $(\mathrm{cN}+)$ and $47.3 \%(n=43 / 91)$ of patients had pathological evidence nodal metastasis $(\mathrm{pN}+)$. Comparing $\mathrm{cN}$ and $\mathrm{pN}, 41.8 \%(\mathrm{n}=38 / 91)$ of cases were congruent, $23.1 \%$ ( $n=21 / 91$ ) were pathologically downstaged and $35.2 \%$ ( $n=$ 32/91) were pathologically upstaged. The average number of pathologically positive nodes per patient was 1.98 (range 


\section{Graph 1: Clinical vs. pathological nodal status.}

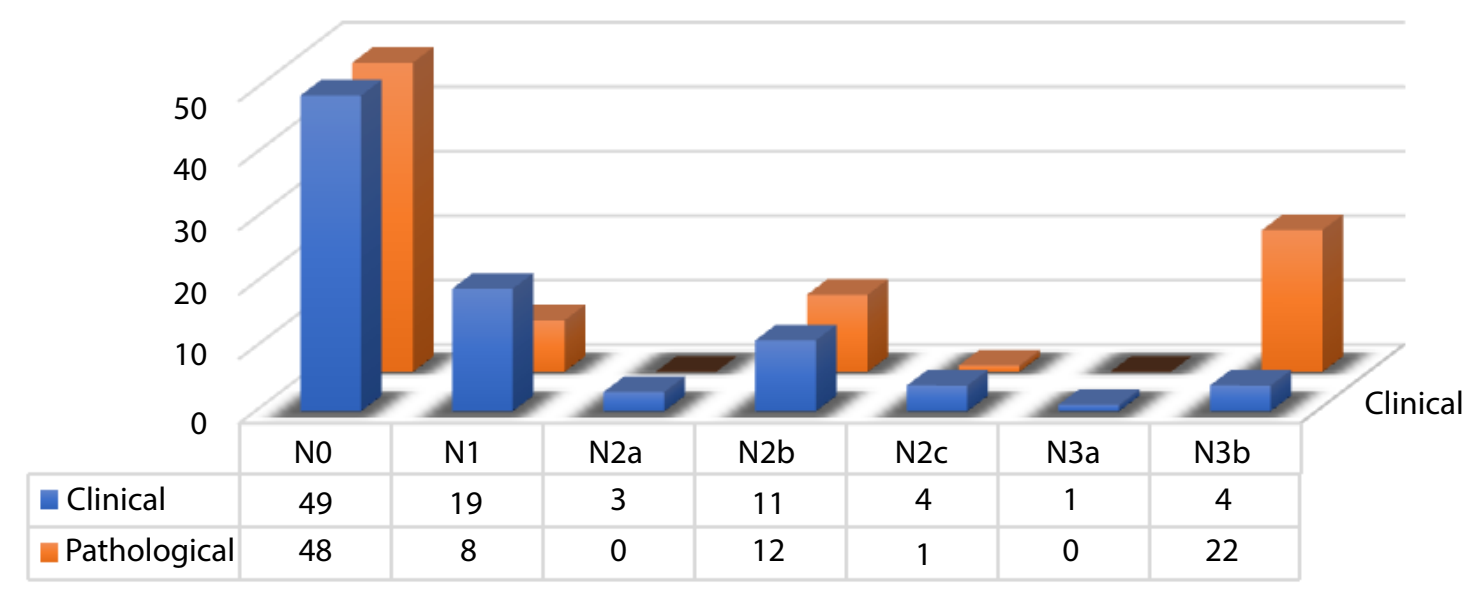

- Clinical Pathological

Table 1: Patient and tumour factors.

\begin{tabular}{|c|c|c|}
\hline Variables & & n (\%) \\
\hline \multirow{2}{*}{ Sex } & Male & $61(67.1)$ \\
\hline & Female & 30 (32.9) \\
\hline \multirow{3}{*}{ Age } & Mean & 57.3 \\
\hline & Youngest & 5 \\
\hline & Eldest & 85 \\
\hline \multirow{3}{*}{ Smoking history } & Present & $67(73.6)$ \\
\hline & Absent & $13(14.3)$ \\
\hline & Unknown & 11 (12.1) \\
\hline \multirow{6}{*}{ Anatomical subsite } & Oral tongue & 35 (38.4) \\
\hline & Floor of mouth & 27 (29.7) \\
\hline & Alveolus & 16 (17.6) \\
\hline & Retromolar trigone & 9 (9.9) \\
\hline & Buccal mucosa & $2(2.2)$ \\
\hline & Hard palate & $2(2.2)$ \\
\hline \multirow{9}{*}{ Histological subtype } & $\begin{array}{l}\text { Conventional } \\
\text { (Unknown } \\
\text { differentiation) }\end{array}$ & $9(9.9)$ \\
\hline & $\begin{array}{l}\text { Conventional (Well } \\
\text { differentiated) }\end{array}$ & $1(1.1)$ \\
\hline & $\begin{array}{l}\text { Conventional } \\
\text { (Moderately } \\
\text { differentiated) }\end{array}$ & $18(19.8)$ \\
\hline & $\begin{array}{l}\text { Conventional (Poorly } \\
\text { differentiated) }\end{array}$ & $57(62.6)$ \\
\hline & Basaloid & $1(1.1)$ \\
\hline & $\begin{array}{l}\text { Carcinoma } \\
\text { cuniculatum }\end{array}$ & $1(1.1)$ \\
\hline & Papillary & $1(1.1)$ \\
\hline & Anaplastic & $1(1.1)$ \\
\hline & Acantholytic & $2(2.2)$ \\
\hline
\end{tabular}

$0-16)$. The average number of pathologically positive nodes in the $\mathrm{pN}+$ subgroup was 4.21 per patient (range 1-16). A total of $24.2 \%$ ( $n=22 / 91)$ patients had pathologically confirmed ENE.

The relationship between $\mathrm{cN}$ and $\mathrm{pN}$ for different nodal stages are demonstrated in (Graph 1 and Table 2). The cNO subgroup $(n=49)$ showed 19 patients $(38.8 \%)$ harboured occult nodal metastasis. Of these, $47.4 \%$ ( $n=9 / 19)$ had pathological ENE ( $p E N E$ ) resulting in a maximum upstaging to $p N 3 b$. The cN1 subgroup $(n=19)$ had $52.6 \%(n=10 / 19)$ pathological downstaged. Four cases $(21.1 \%$ ) were confirmed as pN1. Five cases (26.4\%) were pathologically upstaged, of which three were maximally upstaged to pN3b because of ENE. The cN2b subgroup $(n=11)$ had four cases $(36.4 \%)$ without pathological nodal involvement. Two cases (18.2\%) were confirmed as pN2b. Five cases (45.5\%) were maximally upstaged to N3b because of ENE. The cN3b subgroup ( $n=$ 4) had two cases pathologically downstaged (one pN1 and another pN2b) and two cases were confirmed as pN3b. Other results are summarised in (Table 2).

The multivariate analysis for $\mathrm{cN}$ and $\mathrm{pN}$ subgroups was simplified to include $\mathrm{cN}, \mathrm{pN}$ and congruency. Three potential relationships were possible, namely congruent, down-staged, or up-staged. In the cNO subgroup $(n=49) 61.2 \%$ of cases were congruent and $38.8 \%$ were upstaged. In the $\mathrm{cN}+$ subgroup ( $\mathrm{n}$ $=42$ ) $19 \%$ of cases were congruent, $50 \%$ were down-staged and $31 \%$ were up-staged [Graph 2]. Multivariate analysis using chi-square test were not possible due to insufficient sample size.

Further simplification resulted in a bivariate analysis between $\mathrm{cN}$ and $\mathrm{pN}$ status as simply congruent or non- 
Citation: Rabie ER, Schouwstra CM (2021) The Correlation between Clinical and Pathological Lymph Node Status in Oral Squamous Cell Carcinoma. J Oral Cancer Res 4(1):49-56

Table 2: Prevalence of $\mathrm{cN}$ and $\mathrm{pN}$ nodal status.

\begin{tabular}{|c|c|c|c|c|c|c|}
\hline & & \multicolumn{5}{|c|}{ pN } \\
\hline & & NO & N1 & N2b & $\mathrm{N} 2 \mathrm{c}$ & N3b \\
\hline & & n (\%) & n (\%) & n (\%) & n (\%) & n (\%) \\
\hline & NO & $30(61.2)$ & $3(61)$ & $7(14.3)$ & $0(0)$ & $9(18.4)$ \\
\hline & N1 & $10(52.6)$ & $4(21.1)$ & $1(5.3)$ & $1(5.3)$ & $3(15.8)$ \\
\hline & N2a & $2(66.7)$ & $0(0)$ & $0(0)$ & $0(0)$ & $1(33.3)$ \\
\hline \multirow[t]{4}{*}{$\mathrm{cN}$} & N2b & $4(36.4)$ & $0(0)$ & $2(18.2)$ & $0(0)$ & $5(45.5)$ \\
\hline & $\mathrm{N} 2 \mathrm{c}$ & $1(25)$ & $0(0)$ & $1(25)$ & $0(0)$ & $2(50)$ \\
\hline & N3a & $1(100)$ & $0(0)$ & $0(0)$ & $0(0)$ & $0(0)$ \\
\hline & N3b & $0(0)$ & $1(25)$ & $1(25)$ & $0(0)$ & $2(50)$ \\
\hline
\end{tabular}

\section{Graph 2: Distribution of congruent, down-staged and up-staged} according to $\mathrm{cN}$.

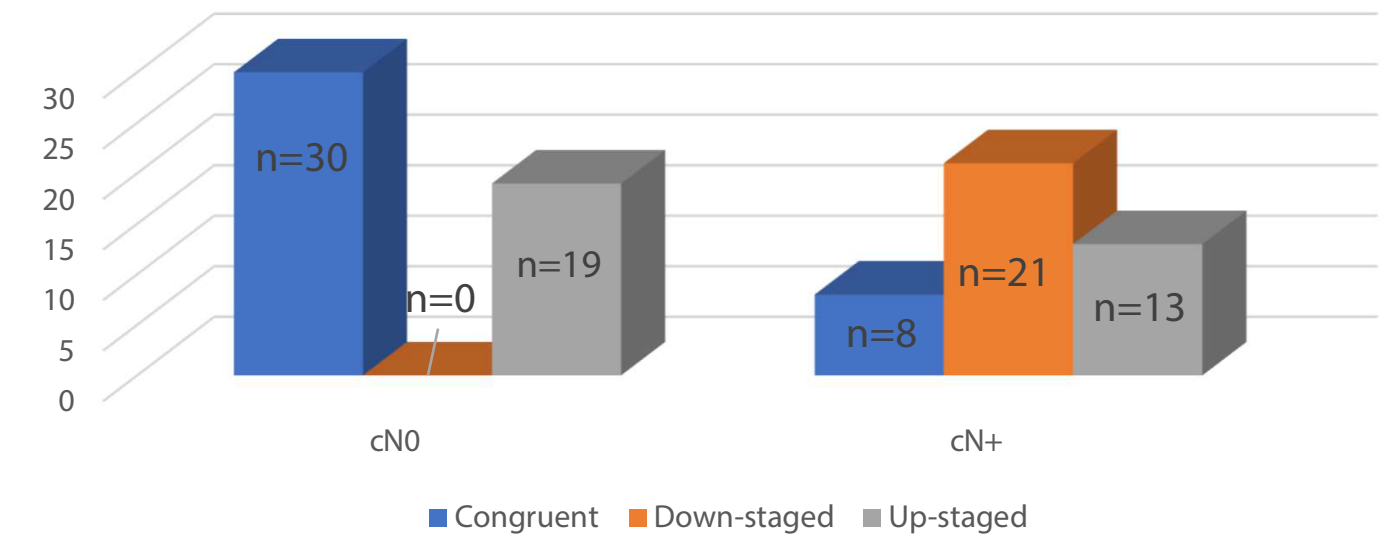

\section{Graph 3: Distribution of pENE according to cN- staging.}

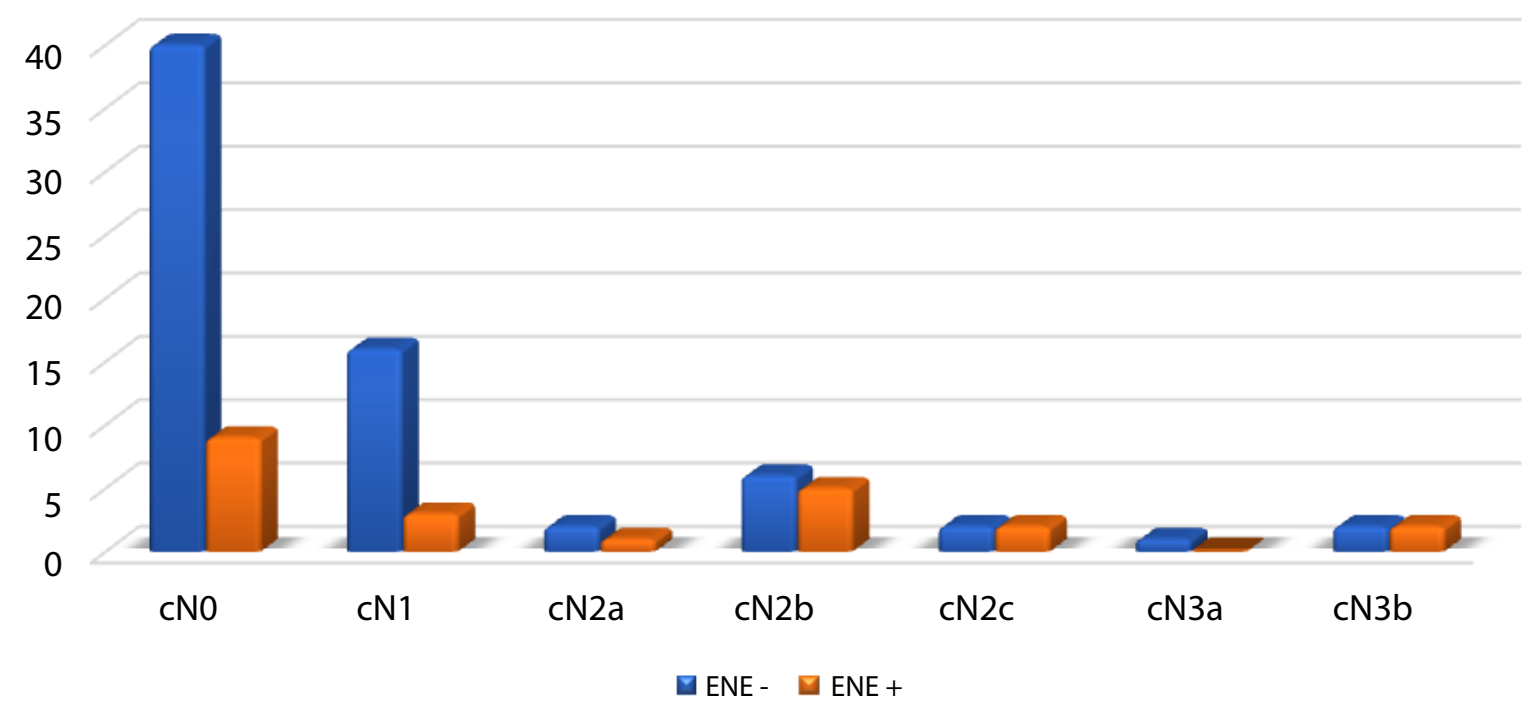


congruent. In the cNO subgroup, $61.2 \%$ of cases were congruent and $38.8 \%$ were non-congruent. In the $\mathrm{cN}+, 57.1 \%$ of cases were congruent and $42.9 \%$ were non-congruent. A chi-square test examined the relation between $\mathrm{cN}$ and $\mathrm{pN}$. The relation between these variables was not significant, $X^{2}(1, N$ $=91)=3.061$. The Fisher exact test statistic value 0.095 was not significant for $p<0.05$. Bivariate statistical analysis supports the hypothesis that the relation between clinical $(\mathrm{cN})$ and pathological $(\mathrm{pN})$ cervical lymph node status in oral squamous cell carcinoma is not statistically significant.

The incidence of pENE the was $24.2 \%(n=22 / 91)$. In the cN0 subgroup, $18.4 \%(n=9 / 49)$ had pENE. Hence, $40.9 \%(n=$ $9 / 22$ ) of pENE cases were found in the cNO subgroup (Graph 3).

\section{Discussion}

Epidemiological studies have examined an array of modifiable and non-modifiable risk factors for OSCC. Males are afflicted twice as often as females in most studies, with this ratio narrowing in recent decades [7]. The current study population consisted of $67 \%$ males and $33 \%$ females. This incidence ratio of nearly 2:1 male to female is similar to what is reported in the literature [2,7-9].

The risk of OSCC increase with age [7]. The median age of our study population was 57 years. This is similar to data from other countries. Surveillance, Epidemiology, and End Results Program (SEER) data from the United States of America between 1996 and 2000 showed a median age of 62 years at the time of oral cancer diagnosis [10]. Data from the Danish national cancer registry between 1984 and 2014 reported a median age of 63 at the time of diagnosis [9]. Interestingly, the age distribution data of our study population showed that $9 \%$ of patients were younger than 45 years of age at the time of diagnosis. This is marginally higher than the $6 \%$ reported in a literature review that included 46 publications devoted to oral cancer in younger cohorts [11].

The aetiopathogenesis of OSCC is multifactorial $[2,12]$. Tobacco smoking is an independent risk factor and the risk is dose dependant [2]. A positive smoking history was present in $73.6 \%$ of the study population, $14.3 \%$ had never smoked and no data on the smoking habits of patients were present in $12.1 \%$. This is similar to reported data that suggest $80 \%$ of OSCC patients has a positive smoking history $[2,12]$. The risk of developing OSCC for smokers is 2-12 times that of nonsmokers [13].

Alcohol has a synergistic effect with tobacco use on the risk of OSCC $[2,12,13]$. A Swedish study found that alcohol consumed at $<10 \mathrm{~g}$ per day did not significantly increase the risk of OSCC [14]. However, alcohol consumed at dosages $>50 \mathrm{~g}$ per day was an independent risk factor for OSCC [14]. Smokers that use alcohol in excess has a 38 times higher risk of developing OSCC, supporting the synergistic effect of the risk factors $[2,14]$.

The most common site for primary tumours were the tongue (38\%) followed by floor of mouth (FOM) (29\%), mandibular alveolus (17\%), retromolar trigone (10\%), buccal mucosa and hard palate ( $2 \%$ respectively). Data from Memorial Sloan Kettering (MSK) Hospital between 1985 and
2015 showed that $51 \%$ of OSCC occur on the tongue, $14 \%$ on the FOM, $14 \%$ on the lower gingiva and $6 \%$ on the RMT [1]. When the combined data of OSCC affecting the tongue, FOM and retromolar trigone at the University of Pretoria and MSK were compared, it was remarkably similar, totalling $77 \%$ and $71 \%$ respectively. When cancers were categorised as either affecting the mandible or the maxilla, a staggering $96 \%$ (Pretoria) and 85\% (MSK) affect the mandible.

This study showed that the relationship between clinical and pathological nodal staging in OSCC was not statistically significant. The data showed that $41.7 \%$ of patients had concordant clinical and pathological nodal staging, 35.2\% where upstaged and $23.1 \%$ where downstaged (Graph 2).

The $41.8 \%$ concordance found in the current study was lower than the congruency rate between $\mathrm{cN}$ and $\mathrm{pN}$ reported in literature $[4,15,16]$. A superficial perusal of the study results may incline the reader to think that a correlation between $\mathrm{cN}$ and pN exists. This is because cNO comprised $53.8 \%$, and the corresponding pNO comprised $52.7 \%$ of the study sample. Similarly, the $\mathrm{cN}$ positive and $\mathrm{pN}$ positive consisted of $46.2 \%$ and $47.3 \%$ respectively. An analysis showed that $41.8 \%$ of patients had $\mathrm{cN}$ and $\mathrm{pN}$ data that corresponded. The greatest discordance where between $\mathrm{cN3b}(n=4)$ and $\mathrm{pN} 3 \mathrm{~b}$ ( $n=22$ ) subgroups. In a retrospective study by Greenberg and colleagues, it was found that $54.4 \%$ of $\mathrm{cN}$ and $\mathrm{pN}$ stages were congruent among $\mathrm{cNO}, \mathrm{cN} 1$ and $\mathrm{cN} 2 \mathrm{a}$ patients [4]. The congruency was highest among the cNO subgroup with $66.1 \%$, followed by cN1 with $25.5 \%$ and $0 \%$ among cN2a patients [4].

A similar trend towards poorer $\mathrm{cN}$ and $\mathrm{pN}$ congruency for more advanced disease cannot be confirmed in the current study due to insufficient sample size. Kreppel, et al. retrospectively reviewed the clinical and pathological data of 392 patients with OSCC [15] and found 59\% concordance between $\mathrm{cN}$ and pN [15]. In discordant cases, 86\% were pathologically downstaged whereas $14 \%$ were upstaged [16]. This represents a higher rate of clinical overestimation compared with the current study which found that $39.6 \%$ ( $n=$ $21 / 53$ ) of discordant cases where clinically over staged.

The poor correlation can potentially be explained by two factors. Patients with clinically evident lymph nodes could be upstaged when considering the incorporation of ENE to the AJCC $8^{\text {th }}$ editions' CTNM staging system. Secondly, it is welldocumented that ENE can present in sub-centimetre lymph nodes and that the lower limit of nodal palpation is $0.5 \mathrm{~cm}$ for superficial and $1 \mathrm{~cm}$ for deeper lymph nodes [4,17]. Therefore, some cNO or cN1 necks might harbour nodal disease with ENE which are clinically undetectable due to the small size and deep cervical location of the involved nodes.

A quarter of clinically occult metastases are too small to be detected by available imaging techniques [18].Van den Brekel was the first to validate radiologic criteria for identification of cervical nodal metastasis [19]. The three variables that was found to be significantly associated with nodal disease are axial diameters of $11 \mathrm{~mm}$ and more for jugulodigastric nodes or $10 \mathrm{~mm}$ and more for any other cervical lymph nodes, groups of three or more borderline lymph nodes and central necrosis of lymph nodes [19]. 
Citation: Rabie ER, Schouwstra CM (2021) The Correlation between Clinical and Pathological Lymph Node Status in Oral Squamous Cell Carcinoma. J Oral Cancer Res 4(1):49-56

The transverse to axial ratio of more than 2 predict nodes involved by metastasis [20]. Aiken and colleagues reported that pre-operative CT evaluation identified central necrosis in $84 \%$ of patients with histologically confirmed ENE, whereas $7 \%$ of patients without histologically confirmed ENE had central nodal necrosis [21]. It has been reported that $40 \%$ of pathologically confirmed nodal metastases are less than $7 \mathrm{~mm}$ in diameter, [22] while ENE has been demonstrated in nodes as small as $2 \mathrm{~mm}$ in diameter [17]. This highlights the fact that by the time radiographic evidence of nodal metastasis becomes apparent, nodal disease is frequently well-established. Hence, once radiographic evidence of nodal metastasis is identified, the likelihood of nodal metastasis is extremely high (high specificity), but the absence of radiographic evidence does not exclude occult nodal metastasis (low sensitivity). The implication is that radiographic evidence of nodal disease would necessitate the surgeon to perform a therapeutic neck dissection, but negative radiographic findings do not aid the surgeon in determining the need for cervical lymphadenectomy. The need for an elective neck dissection in this setting is dependent on clinical and histological factors associated with the primary tumour [22-25].

Of concern, $35.2 \%$ of patients where pathologically upstaged. Significantly, 38.8\% ( $n=19 / 49)$ of the cNO subgroup where upstaged and $47.4 \%(n=9 / 19)$ of these had pENE resulting in maximal upstaging to $\mathrm{pN} 3 \mathrm{~b}$. This rate of occult nodal metastasis is similar to what is reported in the literature $[1,4,22,26]$. It is significant for two reasons.

Patients with OSCC and cNO neck are offered either observation of the neck (in small primary tumours) or a selective cervical lymphadenectomy procedure involving levels I-III [1,13,27] which could potentially result in incomplete regional disease clearance. Although the indication to proceed with a neck dissection in a cNO are influenced by tumour factors such as T-stage and anatomical subsite, the absence of clinically evident lymph nodes does influence the extent of neck dissection. Shah et al retrospectively assessed the patterns of nodal metastasis in 501 OSCC patients [5]. They found that skip metastasis for oral cavity cancer to level IV and $\mathrm{V}$ was $3 \%$ and $1.5 \%$ respectively [5]. Level IV and $\mathrm{V}$ was affected in $17 \%$ and $8 \%$ respectively, when nodal disease was present in the first-echelon nodes (levels I-III) for oral cavity cancers [5]. Hence, the risk of nodal metastasis in levels IV and $V$ significantly increases only when first-echelon lymph nodes harbour metastatic disease.

Secondly, the 5-year survival rate of patients with OSCC and PENE diminishes by approximately half compared with metastatic disease confined to lymph nodes $[26,28]$. Whether these factors had prognostic significance in our cohort remains unknown, as the study database lacked survival data. The prognostic significance of a selective neck dissection (SND) and pENE status in upstaged cNO subgroup requires a prospective controlled investigation. Recent review articles suggest that locoregional control rates in patients undergoing SND is non-inferior compared with modified radical neck dissection (MRND) and appears oncologically sound [ 22,29].

A systematic review by Rodrigo et al reported that current literature supports the oncological safety of SND in patients with cN1 and cN2 disease [29]. Rodrigo and colleagues concluded that selection of candidates for SND must include cN1 and cN2 disease less than $3 \mathrm{~cm}$ in diameter, no palpable nodal metastasis in levels IV or V and no cENE [29]. There are weaknesses in the systematic review as it includes data from three retrospective studies and some studies report unacceptability high regional failure rates that might indicate undertreatment. Studies have shown that $\mathrm{pN}+$, three or more involved nodes, and involvement of levels IV and $\mathrm{V}$ were independent predictors of poor survival [30,31]. Pragmatic thinking would suggest more aggressive surgical treatment or novel treatment strategies would improve locoregional control and survival.

A group of $23.1 \%(n=21)$ was pathologically downstaged in the current study. As pathologically upstaged patients might be offered insufficient cervical lymphadenectomy, patients that are pathologically downstaged could have received unnecessary surgical intervention. Of the $21 \mathrm{cN}+$ patients that were downstaged, 18 where downstaged to pNO. This is clinically significant as the preferred neck dissection carried out for cNO patients with OSCC is a selective neck dissection (SND) I-III [27]. Instead, these $\mathrm{CN}+$ patients received a modified radical neck dissection (MRND) [27]. A prospective study by Gou, et al. analysed data from 322 patients who either underwent a SND I-III or a MRND for cNO disease [32]. The MRND subgroup had a significantly higher complication rate (13.0\% vs $21.9 \%$ with $P=0.04$ ) [32] Significantly, the regional control rates were better for the SOD subgroup, the 3-year disease specific survival were similar and patients in the SND I-III cohort had lower pain and shoulder dysfunction scores [32]. Subjecting patients to unnecessary surgical intervention is contrary to the principles of "first do no harm". This is prudent in the era of evidence-based practice, where surgical techniques aim to minimise morbidity and more selective lymphadenectomy strategies are becoming the norm.

The incidence of pENE in this study population was $24.2 \%(n=22)$. It is similar to the incidence of pENE in similar population groups $[4,33,34]$. Greenberg and colleagues studied 186 cN0 patients. Of the $34 \%$ of patients with occult metastasis, $19 \%$ had pathological evidence of ENE [4]. This is lower than the $47.4 \%$ of ENE reported among cNO patients with occult metastasis in the current study. Shaw et al reported the incidence of ENE among 400 patients with oral squamous cell carcinoma to be $25 \%$ and Chang et al reported the incidence of ENE among 341 patients to be $17.6 \%$. The current study data suggest that the incidence of pENE steadily increases with severity of clinical nodal status; from $16.3 \%$ (cN0), 15.8\% (cN1), 33.3\% (cN2a), 45.4\% (cN2b) and $50 \%$ (cN2c). Statistical analysis of this trend could not be performed due to insufficient sample size.

The retrospective study design is a weakness as potential study participants were excluded due to insufficient data in the clinical and/or pathological records. The University of Pretoria oral cancer database was retrospectively developed. Despite including all available OSCC cases at the University of Pretoria within the study period, the limited sample size in the study is a weakness. Chi-Square analysis becomes unreliable when data elements in subgroups are less than 5, which was 
the case with the multivariate analysis. Tumour factors that influence risk of nodal metastasis include anatomical subsite, DOI [23] and ploidy status $[35,36]$ were not considered as cofounding variables.

\section{Conclusion}

Clinical palpation of the neck has a poor correlation with pathologically confirmed nodal disease in patients with OSCC. The presence of nodal metastasis in first-echelon lymph nodes increases the risk of harboring nodal disease in more distal nodes. More than a third of $\mathrm{cNO}$ patients harbor occult metastasis and the standard of care procedure, a SND I-III might leave behind residual disease in levels IV and V in this cohort. Conversely, patients that are pathologically downstaged to pNO would have received the standard of care MRND procedure with no therapeutic benefit but the associated morbidity. Every effort should be made to improve the accuracy of pre-operative assessment of the cervical nodal basin.

\section{Conflict of Interest}

The authors report no conflict of interest.

\section{Ethics Statement/Confirmation of Patients' Permission}

The study was conducted following approval by the Research Ethics Committee (Reference No. 330/2020) in terms of the National Health Act (Act 61 of 2003), the Code of Ethics for Research of the University of Pretoria and the National Health Research Ethics Council. All research activities were conducted in accordance with the Helsinki declaration. The materials and methodology employed in this study conform to those in currently published literature and thus facilitate reliable reproduction while maintaining patient anonymity. The principles of informed consent and the appropriate wavering thereof were adhered to.

\section{Acknowledgements}

Thank you to Professor Corne Postma from the Department of Dental Management Sciences, University of Pretoria, for statistical support.

\section{References}

1. Shah JP, Patel SG, Singh B, et al. (2012) Jatin Shah's Head and Neck Surgery and Oncology E-Book. Elsevier Health Sciences.

2. Warnakulasuriya S (2009) Global epidemiology of oral and oropharyngeal cancer. Oral oncology 45: 309-316.

3. Abu-Ghanem S, Yehuda M, Carmel N-N, et al. (2016) Elective neck dissection vs observation in early-stage squamous cell carcinoma of the oral tongue with no clinically apparent lymph node metastasis in the neck: A systematic review and metaanalysis. JAMA Otolaryngology-Head \& Neck Surgery142: 857865.

4. Greenberg JS, El Naggar AK, Mo V, et al. (2003) Disparity in pathologic and clinical lymph node staging in oral tongue carcinoma: Implications for therapeutic decision making. Cancer: Interdisciplinary International Journal of the American Cancer Society 98: 508-515.
5. Shah JP, Candela FC, Poddar AK, et al. (1990) The patterns of cervical lymph node metastases from squamous carcinoma of the oral cavity. cancer 66: 109-113.

6. Brierley JD, Gospodarowicz MK,Wittekind C, et al. (2017) TNM classification of malignant tumours. John Wiley \& Sons.

7. Warnakulasuriya S. (2010) Living with oral cancer: Epidemiology with particular reference to prevalence and life-style changes that influence survival. Oral oncology 46: 407-410.

8. Bray F, Ferlay J, Soerjomataram I, et al. (2018) Global cancer statistics 2018: GLOBOCAN estimates of incidence and mortality worldwide for 36 cancers in 185 countries. CA: A cancer journal for clinicians 68: 394-424.

9. Karnov KKS, Grønhøj C, Jensen DH, et al. (2017) Increasing incidence and survival in oral cancer: A nationwide Danish study from 1980 to 2014. Acta Oncologica 56: 1204-1209.

10. Gloeckler Ries LA, Reichman ME, Lewis DR, et al. (2003) Cancer survival and incidence from the Surveillance, Epidemiology, and End Results (SEER) program. The oncologist 8: 541-552.

11. Llewellyn C, Johnson N,Warnakulasuriya K et al. (2001) Risk factors for squamous cell carcinoma of the oral cavity in young people-a comprehensive literature review. Oral oncology 37: 401-418.

12. Neville BW, Damm DD, Allen CM, et al. (2015) Oral and maxillofacial pathology. Elsevier Health Sciences.

13. Peterson $\sqcup$ (2012) Peterson's principles of oral and maxillofacial surgery. PMPH-USA.

14. Lewin F, Norell SE, Johansson H, et al. (1998) Smoking tobacco, oral snuff, and alcohol in the etiology of squamous cell carcinoma of the head and neck: A population-based case-referent study in Sweden. Cancer: Interdisciplinary International Journal of the American Cancer Society 82: 1367-1375.

15. Kreppel M, Nazarli P, Grandoch A, et al. (2016) Clinical and histopathological staging in oral squamous cell carcinomaComparison of the prognostic significance. Oral oncology 60: 68-73.

16. Van den Brekel M, Castelijns J, Stel H, et al. (1993) Modern imaging techniques and ultrasound-guided aspiration cytology for the assessment of neck node metastases: A prospective comparative study. European archives of oto-rhino-laryngology 250: 11-17.

17. Don DM, Calcaterra TC, Anzai Y, et al. (1995) Evaluation of cervical lymph node metastases in squamous cell carcinoma of the head and neck. The Laryngoscope 105: 669-674.

18. van den Brekel MW, Castelijns JA, Snow GB (1998) Diagnostic evaluation of the neck. Otolaryngologic Clinics of North America 31: 601-620.

19. Brekel MWvd, Stel HV, Castelijns JA, et al. (1990) Cervical lymph node metastasis: Assessment of radiologic criteria. Radiology 177: 379-384.

20. Seeburg DP, Baer AH, Aygun N et al. (2018) Imaging of patients with head and neck cancer: From staging to surveillance. Oral and Maxillofacial Surgery Clinics 30: 421-433.

21. Aiken A, Poliashenko S, Beitler J, et al. (2015) Accuracy of preoperative imaging in detecting nodal extracapsular spread in oral cavity squamous cell carcinoma. American Journal of Neuroradiology 36: 1776-1781.

22. Afzali P, Ward BB (2019) Management of the neck in oral squamous cell carcinoma: Background, classification, and current philosophy. Oral and Maxillofacial Surgery Clinics 31: 69-84. 
23. Brockhoff HC, Kim RY, Braun TM, et al. (2017) Correlating the depth of invasion at specific anatomic locations with the risk for regional metastatic disease to lymph nodes in the neck for oral squamous cell carcinoma. Head \& neck 39: 974-979.

24. Dias FL, Kligerman J, De Sá GM, et al. (2001) Elective neck dissection versus observation in stage I squamous cell carcinomas of the tongue and floor of the mouth. Otolaryngology-Head and Neck Surgery 125: 23-29.

25. Shah JP, Gil Z (2009) Current concepts in management of oral cancer-surgery. Oral oncology 45: 394-401.

26. Woolgar JA, Triantafyllou A, Lewis JS, et al. (2013) Prognostic biological features in neck dissection specimens. European Archives of Oto-Rhino-Laryngology 270: 1581-1592.

27. Network NCC (2018) NCCN Clinical Practice Guidelines in Oncology NCCN Guidelines: Head and Neck Cancers Version 2.2018.

28. Ettinger KS, Ganry L, Fernandes RP et al. (2019) Oral cavity cancer. Oral and Maxillofacial Surgery Clinics 31: 13-29.

29. Rodrigo JP, Grilli G, Shah JP, et al. (2018) Selective neck dissection in surgically treated head and neck squamous cell carcinoma patients with a clinically positive neck: Systematic review. European Journal of Surgical Oncology 44: 395-403.

30. Gourin CG, Conger BT, Porubsky ES, et al. (2008) The effect of occult nodal metastases on survival and regional control in patients with head and neck squamous cell carcinoma. The Laryngoscope 118: 1191-1194.
31. de Juan J, García J, López M, et al. (2013) Inclusion of extracapsular spread in the pTNM classification system: A proposal for patients with head and neck carcinoma. JAMA otolaryngology-head \& neck surgery 139: 483-488.

32. Guo CB, Feng Z, Zhang JG, et al. (2014) Supraomohyoid neck dissection and modified radical neck dissection for clinically node-negative oral squamous cell carcinoma: A prospective study of prognosis, complications and quality of life. Journal of Cranio-Maxillofacial Surgery 42: 1885-1890.

33. Chang W-C, Chang C-F, Li Y-H, et al. (2019) A histopathological evaluation and potential prognostic implications of oral squamous cell carcinoma with adverse features. Oral oncology 95: 65-73.

34. Shaw RJ, Lowe D, Woolgar JA, et al. (2010) Extracapsular spread in oral squamous cell carcinoma. Head \& Neck: Journal for the Sciences and Specialties of the Head and Neck 32: 714-722.

35. Hemmer J, Thein T, Van Heerden WF, et al. (1997) The value of DNA flow cytometry in predicting the development of lymph node metastasis and survival in patients with locally recurrent oral squamous cell carcinoma. Cancer: Interdisciplinary International Journal of the American Cancer Society 79: 23092313.

36. Willie FP, van Heerden, Andre W, van Zyl,et al. (2009) Surgical pathology of oral cancer. Diagnostic histopathology 15: 296302.

DOI: $10.36959 / 915 / 578$

Copyright: (C) 2021 Rabie ER. This is an open-access article distributed under the terms of the Creative Commons Attribution License, which permits unrestricted use, distribution, and reproduction in any medium, provided the original author and source are credited. 